\title{
GAIL: Geometry-aware Automatic Image Localization
}

\author{
Luca Benedetti, Massimiliano Corsini, Matteo Dellepiane, Paolo Cignoni, and Roberto Scopigno \\ Visual Computing Lab, ISTI-CNR - Pisa, Italy. \\ E-mail: \{benedetti,corsini,dellepiane,cignoni,scopigno\}@isti.cnr.it
}

Keywords: Image-based Localization, 2D/3D Registration

\begin{abstract}
The access and integration of the massive amount of information, that can be provided by the web, can be of great help in a number of fields, including tourism and advertising of artistic sites. A "virtual visit" of a place can be a valuable experience before, during and after the experience on-site. For this reason, the contribution from the public could be merged to provide a realistic and immersive visit of known places. We propose an automatic image localization system, which is able to recognize the site that has been framed, and calibrate it on a pre-existing 3D representation. The system is characterized by very high accuracy and it is able to validate, in a completely unsupervised manner, the result of the localization. Given an unlocalized image, the system selects a relevant set of pre-localized images, performs a Structure from Motion partial reconstruction of this set and then obtain an accurate camera calibration of the image with respect to the model by minimizing distances between projections on the model surface of corresponding image features. The accuracy reached is enough to seamlessly view the input image correctly super-imposed in the $3 \mathrm{D}$ scene.
\end{abstract}

\section{Introduction}

Automatic image localization is an active research field in Computer Vision and Computer Graphics, with many important applications. This has become especially important given the potentials of all the images coming from the web community. Traditional localization solutions, e.g. Global Positioning System (GPS), may present issues in certain urban areas or indoor environments, or may not be accurate enough. Moreover, both the position and the orientation of the camera could be a valuable source of data. Alternatives, like inertial drift-free systems, are too expensive to be applied on a large scale. In this case, the only class of solutions realistically feasible today is the use of image-based localization systems. Many aspects of the automatic image localization problem have been independently tackled, and tremendous advances have been obtained in recent years.

Here, we are interested in the automatic user localization through the use of digital consumer cameras or smartphones to support information services for tourists. In particular, we cope with a particular image localization scenario, that, to our knowledge, has never been faced in literature: exploiting pre-existing high quality 3D models of the photographs' environment for performing an offline, fully automatic, precise, unsupervised image localization. We aim to ob- tain such an accuracy to allow a seamless view immersion into the 3D scene by projecting the photos on the 3D models. High accuracy allows to re-visualize the picture of the tourist in PhotoCloud (Brivio et al., 2012) that is a CG application which shares some similarities with Photo Tourism (Snavely et al., 2006). This is one of the main goal of an ongoing project related to tourism and valorization of artistic sites.

The proposed system effectively merges solutions from image retrieval, Structure from Motion and $2 \mathrm{D} / 3 \mathrm{D}$ registration. In this context, our contribution is twofold: an image-based localization algorithm capable to obtain very high accuracy by exploiting preexisting high quality $3 D$ models of the locations of interest, and an unsupervised validation algorithm that guarantees to present only correct results to the user. The developed system works by exploiting a dataset of pre-aligned digital photographs on 3D models of the locations of interest.

\section{Related work}

Image localization is a vast field. Here, we present a brief overview of some of the most relevant publications.

Morris and Smelyanskiy (Morris and Smelyanskiy, 2001) faced the problem of single image calibra- 
tion over a 3D surface and the simultaneous surface refinement based on additional information given by the image. The algorithm is based on the extraction of image salient points (using Harris detector (Harris and Stephens, 1988)) and employs minimization of an objective function via gradient calculation. The approach works relatively well only when there is a good initial estimate of the surface, moreover it is not scalable.

Shao et al. (Shao et al., 2003) treated the problem of database-based image recognition, by comparing them to a reference image through the use of local salient features that are described independently of possible affine transformations between them.

Wang et al. (Wang et al., 2004) proposed a solution for the Simultaneous Localization And Tracking (SLAM) robotic problem (Smith and Cheeseman, 1986). A database of salient points, extracted from the robot camera, is used for the localization. SLAM approaches suffer from the "Kidnap problem", i.e. the inability to continue the localization and mapping between non-contiguous locations.

Cipolla et al. (Cipolla et al., 2004) tried to solve this by applying wide baseline matching algorithm techniques between a digital photo and a geo-referenced database. The main limitation of this approach comes from the manual construction of the database correspondences between the map and the photos. Robertson and Cipolla (Robertson and Cipolla, 2004) proposed an improvement of it by exploiting the perspective lines relative to the vertical edges of buildings.

Zhang and Kosecka (Zhang and Kosecka, 2006) built a prototype for urban localization of images that relies on a photographic database augmented with GPS information. The system extracts one or more reference images and from these localized the input image.

Paletta et al. (Paletta et al., 2006) defined a specific system devoted to the improvement in the description of the images' salient points, called "informativeSIFT".

Gordon and Lowe (Gordon and Lowe, 2006) proposed the first work which exploited Structure-fromMotion (SFM) for precise localization of the input image. This approach provided interesting ideas in later works (Irschara et al., 2009; Li et al., 2010; Sattler et al., 2011).

Schindler et al. (Schindler et al., 2007) faced the problem of localization in very large datasets of streets' photographs using a tree data structure that indexes the salient features for scalability.

Zhu et al. (Zhu et al., 2008) built another system for large-scale global localization, with very high ac- curacy thanks to the use of 4 cameras, arranged as two stereo pairs.

Xiao et al. (Xiao et al., 2008) proposed a method for the recognition and localization of generic objects from uncalibrated images. The system includes an interesting algorithm for simultaneous localization of objects and camera positions, which combines segmentation techniques, example models and voting techniques. The main purpose of the system is object recognition using structural representation in $3 \mathrm{D}$ space.

Irschara et al. (Irschara et al., 2009) proposed a localization system that effectively exploited imagebased 3D reconstruction. After the reconstruction, each 3D point is associated to a compressed description of the features of the images incidents therein. Such descriptions are indexed using a tree-based vocabularies for efficient searching.

Li et al. (Li et al., 2010) proposed another featurebased approach based on a prioritization scheme. The priority of a point is related to the number of cameras from the reconstruction it is visible in. The use of a reduced set of points of highest priority has several advantages w.r.t. to using all 3D points. This method, in terms of the number of images that can be registered, outperforms the algorithm by Irschara et al.

Recently, Sattler et al. (Sattler et al., 2011) proposed a direct 2D-to-3D matching framework. By associating 3D points to visual words, they quickly identify possible correspondences for 2D features which are then verified in a linear search. The final 2D-to-3D correspondences are then used to localize the image using $N$-point pose estimation.

Our work shares some similarities with the methods of Irschara (Irschara et al., 2009) and Sattler (Sattler et al., 2011). The novelty stands in the use of a more advanced image retrieval algorithm, the exploitation of 3D geometric information that is not dependant on the photographic dataset, and the validation through an unsupervised validation algorithm.

\section{Geometry-aware Automatic Image Localization}

Our system deals with two specific requirements: the localization hast to be automatic and accurate enough to allow correct superimposition of the input image on the 3D model for presentation purposes to the tourists. There are no strict time constraints.

Our solution combines a state-of-the-art image retrieval system, an SFM algorithm and solutions coming from $2 \mathrm{D} / 3 \mathrm{D}$ registration to recast the problem in a large-scale 2D/3D calibration problem. 


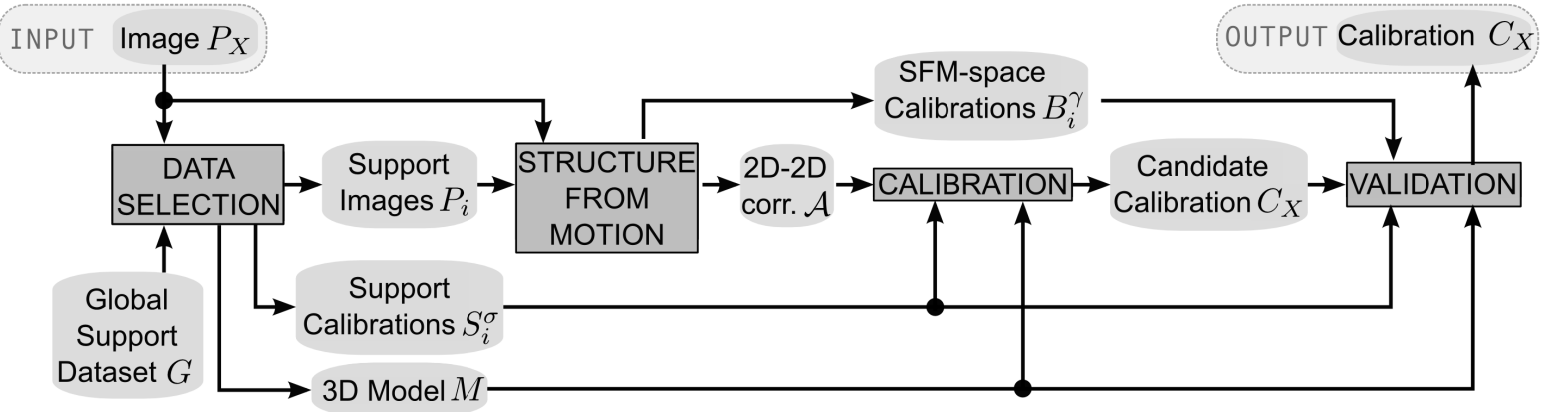

Figure 1: Overview of the algorithm and data flow.

In the following the camera model is defined by 7 parameters: position, orientation, and the focal length. For the intrinsic parameters we assume that the skew factor is zero, the principal point is the center of the images and the scale factors are assumed to be known from the resolution and the CCD dimensions.

\subsection{Overview}

The basic idea of the algorithm is to use an efficient image retrieval system in order to select relevant and local information from the support data (implicitly obtaining a rough approximation of the location), then use such support data to calibrate the camera. Subsequently, the obtained calibration is validated in an unsupervised way to guarantee high accuracy. The data flow is shown in Figure 1.

The input is represented by an image $P_{X}$ that needs to be localized and calibrated. The Data Selection stage takes advantage of a global support dataset $G$. This dataset contains a set of high-resolution 3D models (one for each location of interest), a set of images registered on the respective $3 \mathrm{D}$ model called support images and the corresponding camera parameters.

A retrieval image system is used to obtain a local subset of $G$ composed by the $k$ images $P_{i}$ which are most similar to the input image $P_{X}$. The corresponding support calibration parameters $S_{i}^{\sigma}$ and the 3D model $M$ of the location of interest are also extracted.

The Structure From Motion stage uses $P_{X}$ and the support images $P_{i}$ to perform a Structure from Motion (SFM) algorithm to obtain 2D-2D image correspondences $\mathcal{A}$ and auxiliary camera calibrations $B_{i}^{\gamma}$ (in a coordinate reference system $\gamma$ which is generally different from the reference system of the support 3D model, $\sigma$ ).

Calibration uses the 2D-2D image correspondences (computed at the previous stage), the 3D model $M$ and the support calibrations $S_{i}^{\sigma}$ to calculate a candidate calibration $C_{X}$ of the input image.

Finally, the Validation stage uses the auxiliary camera calibrations $B_{i}^{\gamma}$, the 3D model $M$ and the support calibrations $S_{i}^{\sigma}$ to validate $C_{X}$.

\subsubsection{Creation of the global support dataset}

Concerning the creation of $G$, for each location of interest a set of images that covers as much as possible the model surface is acquired through a photographic campaign. Then, Bundler SFM tool (Snavely et al., 2006) is used to produce an initial camera calibration of the images for each location, including a corresponding point cloud.

Since the results lie on a 3D frame coordinate system that is generally different from the coordinates frame $\sigma$ of the 3D model, we align the 3D points using Meshlab (Cignoni et al., 2008). We obtain a similarity matrix $\Theta$ that brings the set of calibrated images (and the calibrated data) in the $\sigma$ reference system. The user can remove bad calibrated images or attempt to adjust slightly wrong calibrated images by launching the fine alignment registration algorithm implemented in Meshlab. If some area of interest is not covered more images can be added to the set; in this case the Bundler SFM tool has to be re-launched on the expanded dataset.

\subsection{Data Selection stage}

In this stage, Amato and Falchi (Amato and Falchi, 2010) image classifier is used to obtain the subset $\left\{P_{i}\right\}$ of the global support images. The subset is composed by the $k$ images which are classified as the most similar to $P_{X}$. This ensures the scalability of the system since this image retrieval algorithm is able to work very efficiently for ten of thousands of images. The algorithm performs a $\mathrm{kNN}$ classification using local 2D features (SIFT (Lowe, 2004)). We remind the reader to the original publication for the details of the algorithm. 


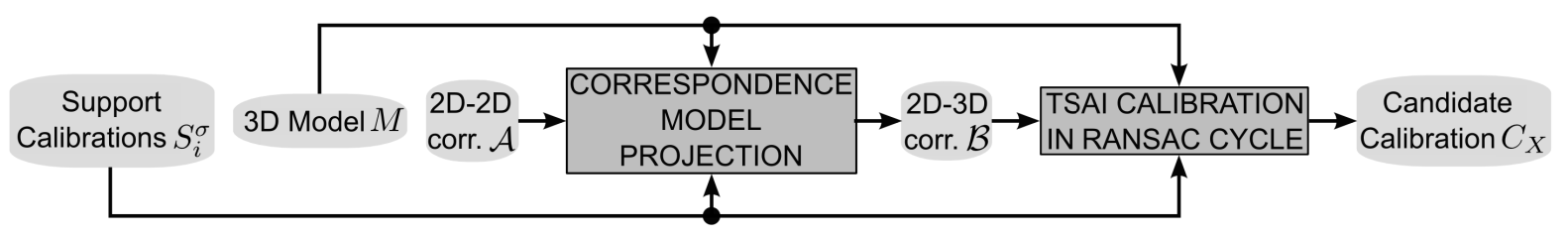

Figure 2: Scheme of the Calibration stage.

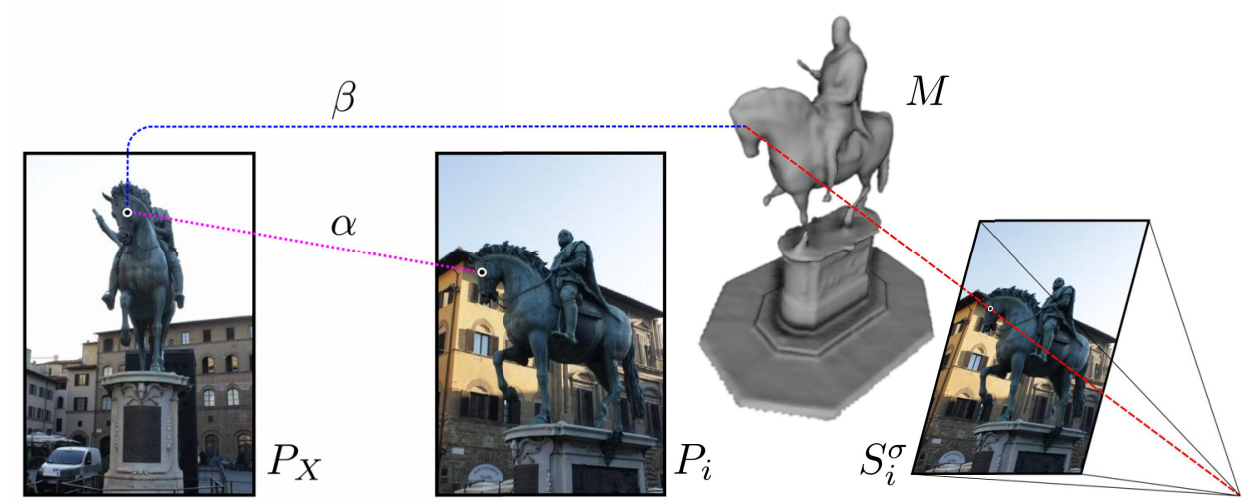

Figure 3: Correspondence projection scheme.

We retrieve a list of 15 similar images $\left(P_{i}\right)$ that form the local support images. This list is further pruned from possible outliers by thresholding over the similarity metric returned in order to have support images that shares at least a partial set of features w.r.t. $P_{X}$. Then, a voting scheme is used to retrieve the 3D model of the location from the set of the available ones. At this point the list is pruned by removing those images that refer to a different 3D model from the one chosen by the voting scheme. If the final list is smaller than a minimal set ( 3 images) the alignment fails and the image is rejected. The local support images just represent a rough approximation of the location of $P_{X}$, since they are associated to a portion of a specific 3D model $M$.

\subsection{Structure From Motion stage}

The local support images together with $P_{X}$ are given in input to Bundler (Snavely et al., 2006) to obtain a set of camera calibrations $B_{i}^{\gamma}$, in a coordinate system $\gamma$, a set of 2D-2D correspondences $\mathcal{A}$ between salient features of the images, and a set of reconstructed 3D points in $\gamma . \mathcal{A}$ is employed in the Calibration stage, $B_{i}^{\gamma}$ are used in the Validation stage.

\subsection{Calibration stage}

The Calibration stage follows the scheme in Figure 2. The goal is to obtain a candidate calibration $C_{X}$ of $P_{X}$ on the 3D model $M$. In order to compute it, we need a set of 2D-3D correspondences $\mathcal{B}$ that matches points on $P_{X}$ with a set of surface points of the 3D model. These correspondences are not known in advance. Nevertheless, we can take advantage of the 2D-2D correspondences $\mathcal{A}$ between the local support images $P_{i}$ and $P_{X}$, and the corresponding calibrations $S_{i}^{\sigma}$, that allow us to project features of $P_{i}$ on the surface of $M$.

The procedure to build $\beta \in \mathcal{B}$ is shown in Figure 3: we project the feature point in $P_{i}$ on the surface of $M$ using the camera calibration $S_{i}^{\sigma}$ and assign the $3 \mathrm{D}$ point with the corresponding $2 \mathrm{D}$ feature point in $P_{X}$. The projection is the intersection between the $3 \mathrm{D}$ surface and the ray connecting the feature point in the image plane with the point of view of the camera. If no intersection is found, no $2 \mathrm{D}-3 \mathrm{D}$ correspondence is generated.

During the construction of $\mathcal{B}$, there are many possible sources of error, such as false positives in $\mathcal{A}$, holes or incongruences between the model and the photographs (i.e. due to movable elements), small errors in camera parameters, etc. Even if multiple 2D2D correspondences of the same visual feature in $P_{X}$ are present, we keep all the possible 2D-3D correspondences. This is because our policy is to keep everything that is potentially correct and to deal with outliers in the following processing step. After obtaining the set of 2D-3D correspondences, we proceed with the effective calibration.

The calibration step follows a RANSAC (Fischler and Bolles, 1981) approach, that in each iteration select a subset of $\mathcal{B}$, making sure to not take duplicates of the same 2D feature on $P_{X}$. Then, it com- 


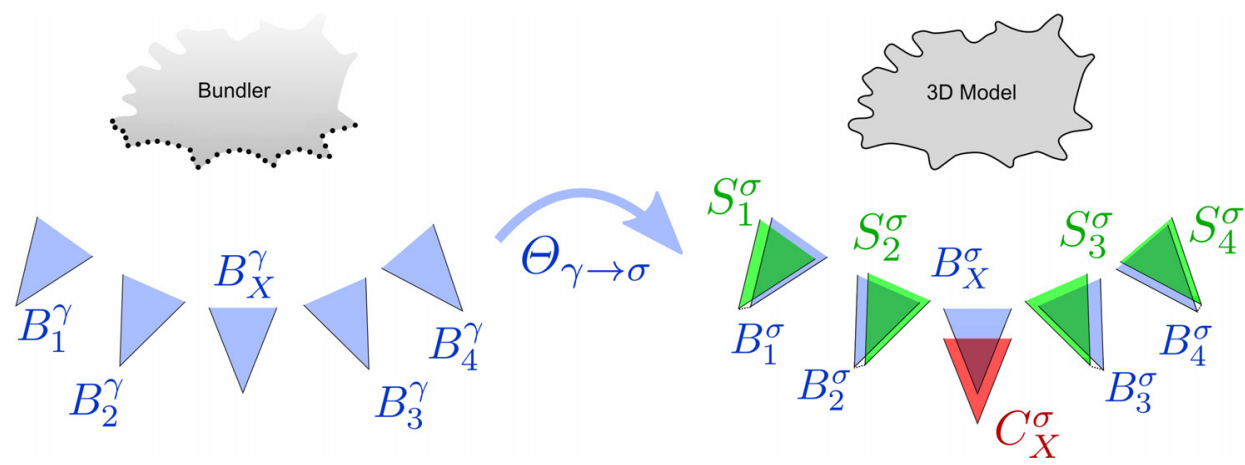

Figure 4: Least Square Mapping scheme.

putes a tentative calibration $C_{X}^{\prime}$ using the well-known Tsai (Tsai, 1987) algorithm, and computes a projection error metric to select the "best" calibration. This approach guarantees robustness with respect to outliers in $\mathcal{B}$. It also has a controlled processing time and avoids "local minima" problems. The RANSAC cycle is limited in time, because the processing time of each iteration is variable and depends on the number of correspondences. The time limit is set to one minute, but we make sure to do between 250 to 1000 iterations.

In each iteration, we randomly sample a constant amount of $202 \mathrm{D}-3 \mathrm{D}$ correspondences $\beta \in \mathcal{B}$ that are used for the calibration with the Tsai algorithm. Tsai calibration works with a minimal amount of 9 correspondences but from our experience we found that 20 correspondences are preferable to obtain good results in presence of noisy data.

After computing the candidate calibration $C_{X}^{\prime}$, we measure its quality. For each $\beta \in \mathcal{B}$, we project its $2 \mathrm{D}$ point on the model surface using $C_{X}^{\prime}$, obtaining the $3 \mathrm{D}$ point $\rho$. If the projection misses the model surface or if the distance of $\rho$ from the 3D point in $\beta$ exceeds a robust threshold we declare a miss, otherwise a success. $C_{X}^{\prime}$ is chosen as the calibration candidate $C_{X}$ if there is any success, the misses are less than $10 \%$ of the total, and the average of the distances in successes is the best one.

\section{Validation stage}

The idea beyond our validation algorithm is to check the consistency between the estimated calibration $C_{X}$ and the calibration parameters provided by the image-based reconstruction done with Bundler (see Section 3.3). Measuring the difference between two camera parameters set is not trivial. We decide to compare what the two cameras are "seeing" in the scene. To calculate such consistency measure, we do the following two steps:

1. We take the calibration $B_{X}^{\gamma}$ given by the imagebased reconstruction for image $P_{X}$ and we map it in our coordinate frame $\sigma$, obtaining $B_{X}^{\sigma}$.

2. We measure how differently $B_{X}^{\sigma}$ and $C_{X}^{\sigma}$ view the same scene comparing two depth maps generated from these data.

To obtain $B_{X}^{\sigma}$, we exploit relationships between the support calibrations $S_{i}^{\sigma}$ and the calibrations $B_{i}^{\gamma}$ computed through Bundler (see Figure 4). The set of cameras have similar geometrical relationships in the camera positions, but differences in estimation are generated, e.g. due to the focal length/view direction ambiguity.

The estimation of the similarity matrix to obtain $B_{X}^{\sigma}$ is performed following a RANSAC approach in order to account for outliers. A subset of the calibrations obtained is selected. The difference in scale is adjusted using the bounding box of the two set of cameras. Then, the Horn's method (Horn, 1987) is applied to estimate a similarity matrix $\Theta_{\gamma \rightarrow \sigma}$ to transform the coordinate frame from $\gamma$ to $\sigma$.

We evaluate the quality of the similarity matrix by applying it to all calibrations $B_{i}^{\gamma}$ and measuring the Euclidean distances between the viewpoints with respect to $S_{i}^{\gamma}$. The most accurate $\Theta_{\gamma \rightarrow \sigma}$ is applied to $B_{X}^{\gamma}$.

In the second stage, we check the consistency of $B_{X}^{\sigma}$ and $C_{X}^{\sigma}$, by doing an image-based comparison on two virtual range maps. We opt for this novel approach since small changes in camera parameters can lead to major differences in the framed area, e.g. due to obstacles.

We proceed by obtaining two low-resolution synthetic range maps $R_{1}$ and $R_{2}$ of the $3 \mathrm{D}$ model as seen by the two cameras obtained. Then, we measure two errors: the XOR consistency $\left(E_{X O R}\right)$ of model occlusion versus the background, and the Sum of Squared Differences (SSD) of depth values $\left(E_{S S D}\right)$ between $R_{1}$ and $R_{2}$. The values of $R_{1}$ and $R_{2}$ are normalized together in the $[0 \ldots 1]$ range. Background values are 


$$
E_{S S D}=\frac{\sum_{X O R}=\frac{\sum_{\substack{0<x<w \\ 0<y<h}} R X\left(R_{1}, R_{2}, x, y\right)}{w h} B H\left(R_{1}, R_{2}, x, y\right) \cdot\left(R_{1}(x, y)-R_{2}(x, y)\right)^{2}}{\sum_{\substack{0<x<w \\ 0<y<h}} B H\left(R_{1}, R_{2}, x, y\right)}
$$
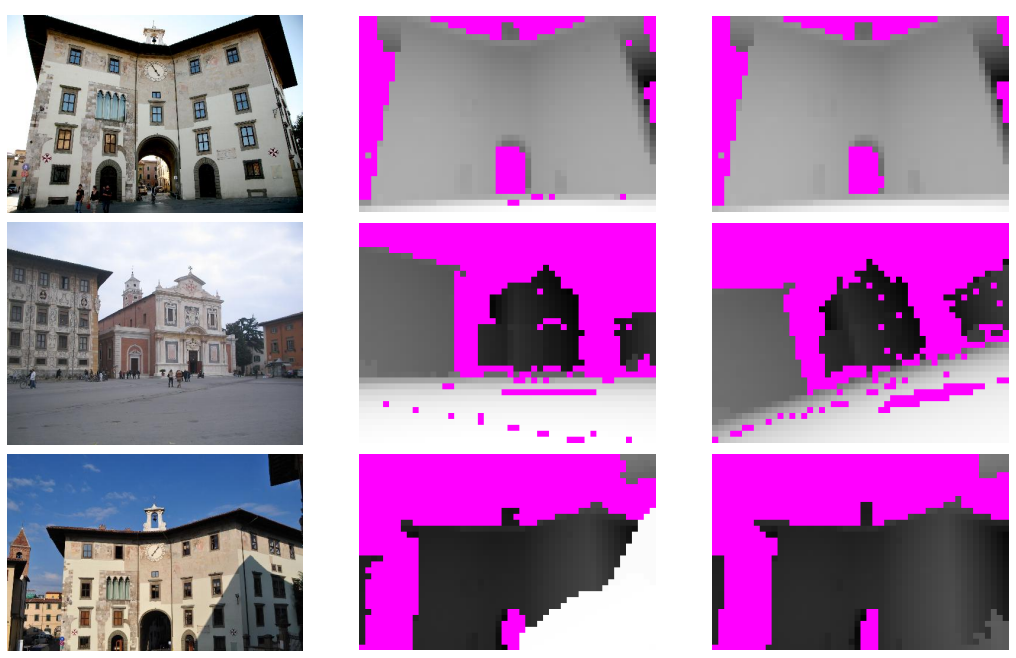

\author{
Almost Correct \\ $E_{X O R}=0.0115$ \\ $E_{S S D}=0.0002$
}

Bad XOR consistency

$E_{X O R}=0.2416$,

$E_{S S D}=0.0349$

\section{Bad SSD error \\ $E_{X O R}=0.0339$ \\ $E_{S S D}=0.1720$}

Figure 5: (Left) Input image. (Center) Range map obtained from $C_{X}^{\sigma}$. (Right) Range map obtained from $B_{X}^{\sigma}$.

set to $\infty$

The XOR consistency (Eq. 1) is the percent of pixels that in $R_{1}$ are background and in $R_{2}$ are not and vice-versa, where $w$ and $h$ are the size of the range maps and $R X\left(R_{1}, R_{2}, x, y\right)$ assumes the value 1.0 when the condition $\left(R_{1}(x, y)=\infty\right) \oplus\left(R_{2}(x, y)=\infty\right)$ is true and 0.0 otherwise. It essentially accounts for different positions and directions of view.

The SSD error (Eq. 2) measures the dissimilarity in non-background areas, where $B H\left(R_{1}, R_{2}, x, y\right)$ assumes the value 1.0 when both the pixels of $R_{1}(x, y)$ and $R_{2}(x, y)$ are foreground pixels and 0.0 otherwise. This measure accounts for errors in position and focal length, that could lead to the different framing of objects which are near to the camera.

Examples of the consistency measures are shown in Figure 5. The second and the third rows show two calibrations which are incorrect due to different reasons: in the first case, the XOR consistency results to be very high; in the second case, the problem is indicated by the value of the SSD error.

\section{Experimental results}

In this section, we will describe and discuss the results of the experimental evaluation of both the full image localization algorithm and the validation step. The global support dataset is composed by images and 3D models for 2 locations: "Piazza Cavalieri" in Pisa (Italy) and "Piazza della Signoria" in Florence (Italy). The "Signoria" location is covered by 304 calibrated images while the "Cavalieri" location by 202 calibrated images. The corresponding 3D models (485k and 4083k faces respectively) have been obtained through ToF laser scanning, and prepared as explained in Section 3.1.1.

\subsection{Comparison with previous work}

In order to assess the performance of our system, we compared it with two recent state-of-the-art works in image localization ( $\mathrm{Li}$ et al., 2010; Sattler et al., 2011). Both these systems were tested using the same "Dubrovnik" dataset ${ }^{1}$, which is composed by 6844 images. The authors test their systems by extracting

\footnotetext{
${ }^{1}$ Available at http://grail.cs.washington.edu/ rome/dubrovnik/index.html
} 
Table 1: Comparison of localization performances between our method and (Li et al., 2010; Sattler et al., 2011).

\begin{tabular}{|c|c|c|}
\hline Tested method & $\begin{array}{c}\text { average localization } \\
\text { error }(\mathrm{m})\end{array}$ & $\begin{array}{c}\text { \# of registered } \\
\text { images }\end{array}$ \\
\hline Li et al (Li et al., 2010) & 18.3 & $94 \%$ \\
Sattler et al (Sattler et al., 2011) & 15.7 & $96 \%$ \\
GAIL (before validation) & 3.9 & $59 \%$ \\
GAIL (after validation) & 2.1 & $26 \%$ \\
\hline
\end{tabular}

800 images from the dataset, and try to localize them. Each test is repeated 10 times.

It was not possible to use the Dubrovnik dataset in our case, because no 3D model of the city is provided, However we applied the same testing approach on our image datasets by attempting to re-align all the pre-calibrated images 10 times. Results are shown in Table 1.

Regarding the localization error, our method outperforms the others. The percentage of acceptance is lower due to the different goal, accurate calibration, of our approach with respect to the goal, localization, of previous work.

Figure 6 shows some examples of the calibrations obtained, we divide the calibration accuracy in: "high quality" (near pixel-perfect superimposition), "medium quality" (small misalignments are present), and "low quality" (severe misalignments with the 3D models or completely wrong result). It has to be noted that several of what we refer as "low quality" alignments could be accepted as correct by a typical localization system where only the position of the camera is important and not the orientation as well. Our goal force us to be more selective to ensure a satisfying navigation of the localized photographs. The thresholds set in the current system implementation, relative to the results here reported, allow for "high quality" calibration.

\subsection{Result evaluation}

In order to evaluate the performance of the system, the validation algorithm in particular, 568 input images were retrieved from Flickr, in order to cover many possible cases that the system must face. These images have been manually inspected before the tests, in order to have an "a priori" knowledge of which ones we expect to locate and which ones we expect to refuse.

This classification is based only on the visual inspection; we consider "not localizable" images that are either relative to part not covered by the 3D model, in very poor lighting condition (i.e. night), or depicting objects that are not strictly part of the scene (e.g. a bicycle, a cup of coffee). See examples in Figure 7.
After this inspection, we expect to accept 319 (56\%) of the 568 images and to refuse the remaining 249. Of these 568 images:

- $180(31.7 \%)$ were rejected in the classification step

- $12(2.1 \%)$ were rejected in the reconstruction step

- $146(25.7 \%)$ were rejected in the calibration step.

This means that 230 images $(40.5 \%)$ were accepted by calibration. Among these:

- 119 (21.0\% of total, $51.7 \%$ of selected) failed the Validation stage.

- 111 (19.5\% of total, $48.3 \%$ of selected) were validated.

The thresholds used for the validation are $E_{X O R} \leq$ 0.15 and $E_{S S D} \leq 0.05$.

The method proves to be very selective, since $38.7 \%$ of the images which were judged to be acceptable were discarded during the first three stages. On the other side, only 2 images ( $0.4 \%$ of the total) were wrongly accepted. This is a key feature for a system which does not need any human-based validation of the results.

Moreover, the datasets which were used were not ideal, both in terms of input data (covering of the support images, quality of 3D model) and type of environment (Piazza della Signoria contains several statues, so that some images depict details which are hard to match due to several occlusions).

We expect that the performance could be improved using a more complete (in terms of coverage) global support dataset.

\subsection{Timing}

Concerning the processing time of the different stages of the system, the time to retrieve the local support set is negligible, since the algorithm by Amato et al. is designed to deal with millions of images, and the global support set is usually composed by hundreds of images. This makes the time to find the similar images practically instantaneous. The calibration stage, as previously stated, is limited to 1 minute (ensuring that a certain number of iterations is reached) in the 


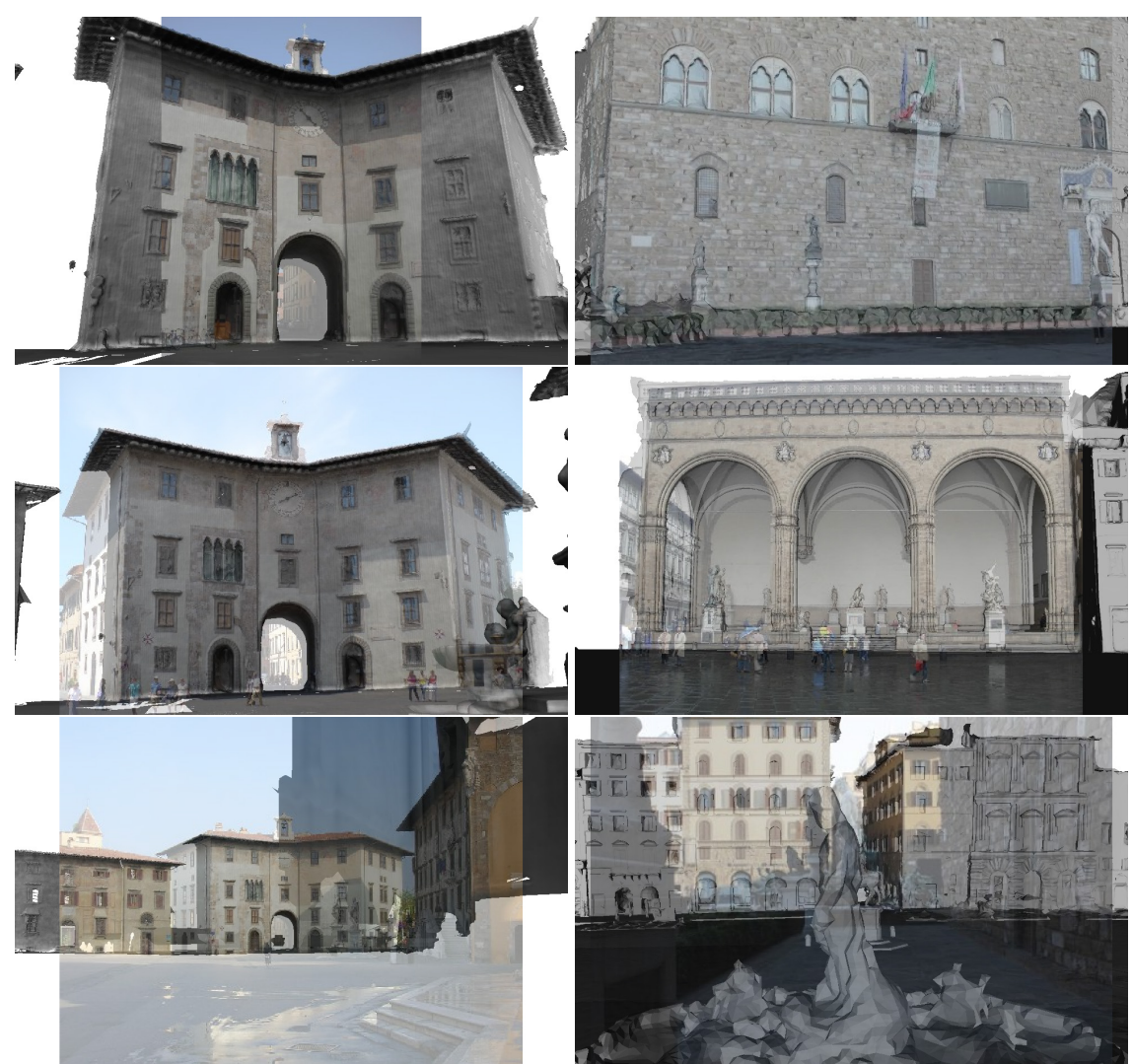

Figure 6: Calibration accuracy examples. (1st Row) High quality. (2nd Row) Medium quality. (3rd Row) Low quality.

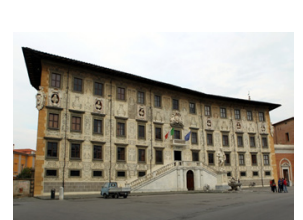

(a) ETL

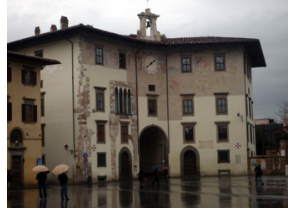

(f) ETL

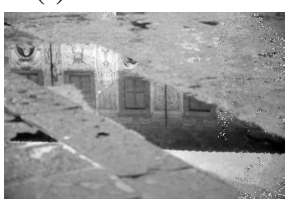

(k) ETR

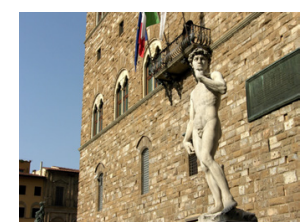

(b) ETL

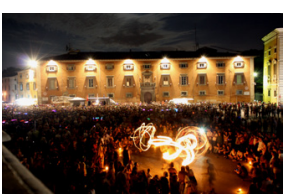

(g) ETR

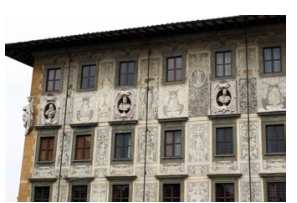

(c) ETL

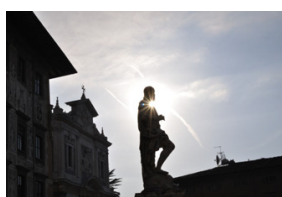

(h) ETR

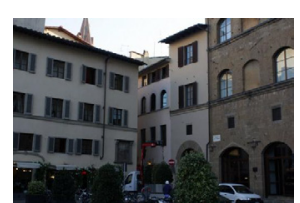

(d) ETL

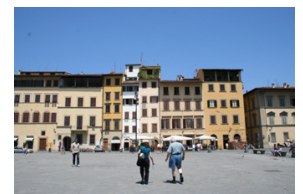

(i) ETR

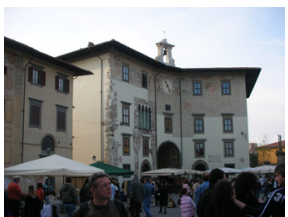

(e) ETL

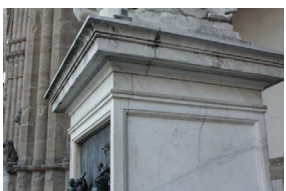

(j) ETR

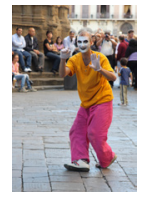

(1) ETR

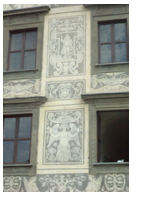

(m) ETR

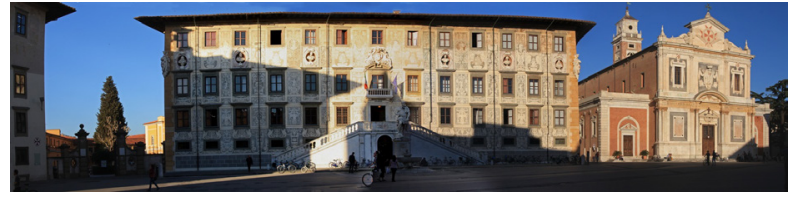

(n) ETR

Figure 7: Examples of images that we expect to locate (ETL): (a) building; (b) statue; (c) partial façade; (d) small clutter; (e) moderate clutter; (f) moderate reflexes. Examples of images that we expect to refuse (ETR): (g) night time; (h) against the light; (i) uncovered area (visually similar to covered areas); (j) small detail of statue; (k) ambiguous detail containing major reflection; (1) major clutter; (m) ambiguous detail; (n) panoramic montage. Note that the image (i), that is visually very similar to a picture of "Piazza della Signoria" in Florence but it is the picture of another plaza, is correctly discarded by the algorithm. 

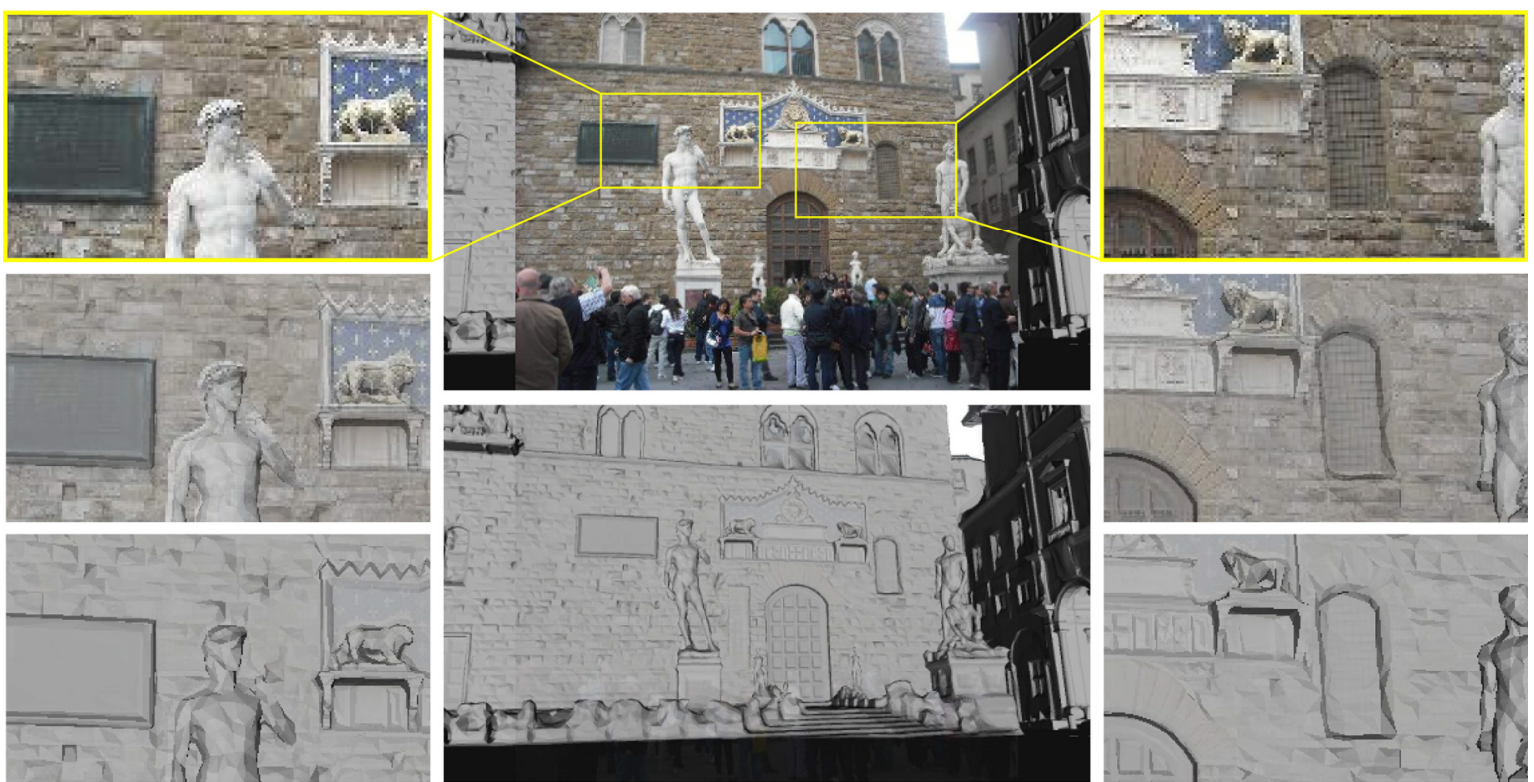

Figure 8: Results example. (Center Top) Input image immersed in 3D. (Center Bottom) 3D model view from $C_{X}^{\sigma}$. (Side Columns) Superimpositions of details.

current implementation, but further optimization to reduce this processing time can be achieved. Finally, the validation stage is quite fast, in the order of tenths of $\mathrm{ms}$ on a average-end PC.

\subsection{Discussion}

The main advantage of the proposed system is that it is able to work in a completely automatic and unsupervised way producing very high accurate camera calibrations for urban context. This implies also a very accurate localization. The selection of images is very strict, in order to ensure the lowest possible rate of false positives.

This also gives the possibility to the system to "train" and increase the robustness, since the successfully calibrated images can be added to $G$ in order to increase the performance of the system itself during its use. Moreover, a very high number of precalibrated images could be used, due to the scalability of the image retrieval algorithm employed.

The main limitation is related to the fact that the validation step discards a calibration more frequently for errors in $B_{X}^{\sigma}$ than for errors in the Calibration stage $C_{X}^{\sigma}$. More research in this direction could be of great interest. Another limitation is that a $3 \mathrm{D}$ model of the scene is needed. Nevertheless, current multiview stereo reconstruction techniques are probably able to provide an accurate enough reconstruction of the scene.

\section{Conclusions}

In this paper we proposed a novel localization algorithm that allows for an accurate 2D/3D registration of the input image in a large scale context, typically an urban context.

An unsupervised validation algorithm of the localization obtained is also proposed. The algorithm is composed of several stages that take advantage of a large amount of information that can be extracted from 2D/3D data: 2D-2D feature correspondences, sparse reconstructions, depth maps from defined points of view.

The performance and the advantages/limitations of the method are assessed and discussed. The system proved to selective, but very accurate and robust. Thus, all the calibrated images could be directly used in a photo navigation system without the need of human validation. Figure 8 shows an example of an image that was perfectly aligned to a complex 3D scene, with objects of different sizes at different distances w.r.t. the point of view.

A possible improvement regards optimizing the system to reduce registration time. Another improvement could be to extract further information from the image to reduce false negatives. Furthermore, the calibration could be further refined before validation. For example, refinement could be obtained using feature matching (Stamos et al., 2008) or statistical (Corsini et al., 2012) methods. 


\section{ACKNOWLEDGEMENTS}

This paper has been supported by the Tuscany Region (POR CREO FESR 2007-2013) in the framework of the VISITO-Tuscany project and by the EU FP7 INDIGO Innovative Training and Decision Support for Emergency Operations project (grant no. 242341). We would also thank Fabio Ganovelli for useful suggestions and insights about this work.

\section{REFERENCES}

Amato, G. and Falchi, F. (2010). kNN based image classification relying on local feature similarity. In Proc. SISAP'10, pages 101-108. ACM.

Brivio, P., Benedetti, L., Tarini, M., Ponchio, F., Cignoni, P., and Scopigno, R. (2012). Photocloud: interactive remote exploration of large 2D-3D datasets. IEEE Computer Graphics and Applications, pages 1-20.

Cignoni, P., Callieri, M., Corsini, M., Dellepiane, M., Ganovelli, F., and Ranzuglia, G. (2008). Meshlab: an open-source mesh processing tool. In Sixth Eurographics Italian Chapter Conference, pages 129-136.

Cipolla, R., Robertson, D., and Tordoff, B. (2004). Imagebased localisation. In Proc. of 10th Int. Conf. on Virtual Systems and Multimedia, pages 22-29.

Corsini, M., Dellepiane, M., Ganovelli, F., Gherardi, R., Fusiello, A., and Scopigno, R. (2012). Fully automatic registration of image sets on approximate geometry. International Journal of Computer Vision, pages 121.

Fischler, M. and Bolles, R. (1981). Random sample consensus: a paradigm for model fitting with applications to image analysis and automated cartography. Communications of the ACM, 24(6):381-395.

Gordon, I. and Lowe, D. (2006). What and where: 3d object recognition with accurate pose. Toward category-level object recognition, pages 67-82.

Harris, C. and Stephens, M. (1988). A combined corner and edge detector. In Alvey vision conference, volume 15. Manchester, UK.

Horn, B. (1987). Closed-form solution of absolute orientation using unit quaternions. JOSA A, (April).

Irschara, A., Zach, C., Frahm, J.-M., and Bischof, H. (2009). From structure-from-motion point clouds to fast location recognition. In CVPR, pages 2599-2606.

Li, Y., Snavely, N., and Huttenlocher, D. P. (2010). Location recognition using prioritized feature matching. In ECCV, pages 791-804.

Lowe, D. (2004). Distinctive image features from scaleinvariant keypoints. International journal of computer vision, 60(2):91-110.

Morris, R. and Smelyanskiy, V. (2001). Matching images to models - camera calibration for 3-d surface reconstruction. Energy Minimization Methods, pages 105117.
Paletta, L., Fritz, G., Seifert, C., Luley, P., and Almer, A. (2006). A mobile vision service for multimedia tourist applications in urban environments. 2006 IEEE Intelligent Transportation Systems Conference, pages 566572.

Robertson, D. and Cipolla, R. (2004). An image-based system for urban navigation. In Proc. BMVC, volume 1, pages 260-272.

Sattler, T., Leibe, B., and Kobbelt, L. (2011). Fast imagebased localization using direct $2 \mathrm{~d}$-to-3d matching. In IEEE International Conference on Computer Vision (ICCV), pages 667-674.

Schindler, G., Brown, M., and Szeliski, R. (2007). City-scale location recognition. In IEEE Conference on Computer Vision and Pattern Recognition (CVPR2007), pages 1-7. IEEE Computer Society.

Shao, H., Svoboda, T., Tuytelaars, T., and Van Gool, L. (2003). HPAT indexing for fast object/scene recognition based on local appearance. CIVR'03, pages $307-$ 312.

Smith, R. and Cheeseman, P. (1986). On the representation and estimation of spatial uncertainty. The international journal of Robotics Research, 5(4):56.

Snavely, N., Seitz, S. M., and Szeliski, R. (2006). Photo tourism: exploring photo collections in 3d. In $S I G-$ GRAPH '06, pages 835-846.

Stamos, I., Liu, L., Chen, C., Wolberg, G., Yu, G., and Zokai, S. (2008). Integrating automated range registration with multiview geometry for the photorealistic modeling of large-scale scenes. Int. J. Comput. Vision, 78:237-260.

Tsai, R. (1987). A versatile camera calibration technique for high-accuracy $3 \mathrm{~d}$ machine vision metrology using off-the-shelf tv cameras and lenses. IEEE Journal of Robotics and Automation, 3(4):323-344.

Wang, J., Cipolla, R., and Hongbin, Z. (2004). Image-based localization and pose recovery using scale invariant features. pages 711-715.

Xiao, J., Chen, J., Yeung, D.-Y., and Quan, L. (2008). Structuring visual words in $3 \mathrm{~d}$ for arbitrary-view object localization. In ECCV' 08 , pages 725-737.

Zhang, W. and Kosecka, J. (2006). Image based localization in urban environments. 3DPVT'06, pages 33-40.

Zhu, Z., Oskiper, T., Samarasekera, S., Kumar, R., and Sawhney, H. (2008). Real-time global localization with a pre-built visual landmark database. In $C V P R$, pages $1-8$. 